\title{
Improving building energy footprint and asset performance using digital twin technology
}

Mohsen A. Jafari PhD

Professor, Department of Industrial and Systems Engineering, Rutgers, the State University of New Jersey, Piscataway, NJ, USA (corresponding author: jafari@soe.rutgers.edu)

\section{Ali Ghofrani PhD}

Adjunct Professor, Department of Industrial and Systems Engineering,

Rutgers, the State University of New Jersey, Piscataway, NJ, USA
Esmat Zaidan PhD

Associate Professor, Department of International Affairs, College of Arts and Sciences, Qatar University Doha, Qatar

Ammar Abulibdeh PhD

Assistant Professor, Department of Humanities, College of Arts and Sciences, Qatar University Doha, Qatar

This article presents a novel architecture by integrating the existing asset management theory with building simulation technology for effective maintenance strategies and operational control schemes. Building performance, value and energy usage collectively define the criteria for optimisation. Building assets are partially or fully connected with building internet of things and their real-time conditions are accessible at all times. An asset's value is derived from the functional contributions of that asset to the overall business objective of the system that it is part of. The architecture consists of digital twin, analytics and business value mode engines and in-between gateways for data exchange. The paper provides illustrative examples for how the platform can serve the operations and maintenance objectives of existing and new buildings.

Keywords: building technology/control/digital twin/O\&M planning/predictive modelling

\section{Notation}

$A_{\mathrm{Eff}_{i}}(h) \quad$ effective age of asset $i$ at time interval $h$

$B^{i} \quad$ lag operator with degree $i$ of the predictive model time series

$\mathrm{CI}_{i} \quad$ condition index of asset $i$

$i \quad$ index of zone number

$j \quad$ index of decision variable

$K \quad$ number of decision variables

$k \quad$ index of maintenance type

$l \quad$ index of frequency

$h \quad$ index of hourly timestep

$h_{\mathrm{w}}(X) \quad$ hypothesis function with model parameter $w$ and feature vector $X$

$n \quad$ size of the training dataset

$P \quad$ number of seasonal autoregressive parameters of predictive model time series

$p \quad$ number of non-seasonal autoregressive parameters of predictive model time series

Q number of seasonal moving average parameters of predictive model time series

$q \quad$ number of non-seasonal moving average parameters of predictive model time series

$R^{t}(i) \quad$ power rate for zone $i$

$\mathrm{RF}_{(T k l)_{i}} \quad$ restoration factor for asset $i$ in maintenance option $(T ; k, l)$

$s \quad$ seasonality factor of predictive model time series

$T \quad$ planning period

$T_{\text {ext }}^{i}(i) \quad$ external temperature for zone $i$

$T_{\text {in }}^{i}(i) \quad$ internal temperature for zone $i$

$t \quad$ index of timestep

$X \quad$ feature vector

$X^{*}$ $w$

$\hat{Y}$

$Y_{i}$

$\hat{Y}_{i}$

$y_{t}$

$\alpha_{i}$

$\beta_{j}$

$\Gamma(w, \alpha)$

$\delta_{i j}$

$\boldsymbol{\delta}(\boldsymbol{T} ; \boldsymbol{k}, \boldsymbol{l})$ assets' performance dependency matrix as result of maintenance option $(T ; k, l)$

$\varepsilon_{t} \quad$ error term of the predictive model time series at timestep $t$

$\varepsilon^{t} \quad$ predictive model error at timestep $t$

$\Theta_{j} \quad j$-th seasonal autoregressive parameter of predictive model time series

$\theta_{i} \quad i$-th non-seasonal moving average parameter of predictive model time series

$\xi_{j}(X) \quad j$ number of arbitrary constraints

$\Pi_{i}(X) \quad i$ number of building physics constraints

$\phi(X) \quad$ cost function

$\phi_{i} \quad$ predictive model parameter of internal temperature for zone $i$

$\phi_{i} \quad i$-th non-seasonal autoregressive parameter of predictive model time series

$\phi_{j} \quad j$-th seasonal autoregressive parameter of predictive model time series

$\Psi(X) \quad$ discomfort function 


\section{Introduction}

The methodology presented here integrates building (facility) digital twin technology with asset condition data and business value to improve building energy efficiency and asset performance and reliability. The methodology takes advantage of both dynamic data, such as the energy-metered data of individual assets, asset condition and performance data, occupancy and static data, such as the business objectives of the building, the functional decomposition of the building and zoning. Assets are (partially or fully) connected (with sensors and embedded devices), and their real-time conditions are accessible at all times. An asset's value is derived from the functional contributions of that asset to the overall business objective of the system that it is part of. Building digital twin runs in parallel to the actual physical building and proactively participates in operations and maintenance (O\&M). The digital twin is expected to reside at a layer above the typical building energy management system (BEMS) with planning time scaled in days or more, and operational time scaled in minutes, hours or longer. The technology can provide solutions to building owners and operators to proactively diagnose faults and failures, run what-if scenarios and benchmarking, optimise building O\&M and carry out planning and resource analysis. In this work, energy consumption is an important asset attribute that is impacted by an asset's degradation and performance.

Building management involves decision making under multiple objectives and uncertainties in addition to budgetary constraints. The existing literature on building asset and energy maintenance and predictive analytics includes works by Lounis and Vanier
(2000), Chin and Gillepsie (2010) and Mahani et al. (2014), among others. There are also commercial asset management systems (e.g. IBM Maximo) for facility managers and real estate executives to manage their assets and reduce operational costs (including the cost of energy). The prioritisation of maintenance actions and budget appropriation are essential to ensure the reliable and efficient performance of assets and to provide continuous building functions without unplanned interruption. The work by Salahi et al. (2013) includes a building value model (BVM) framework that maps business objectives to building assets. The authors' digital twin framework takes advantage of their results.

\section{System architecture}

From a system perspective, a building is defined by its zones and assets that together serve some business functions and objectives. On the other hand, buildings are physics-based objects that interact with their environment in some complex ways. By definition, a digital twin is a computational replica of a physical entity, which exists simultaneously, and seamlessly exchanges data with its physical sibling. Bridging physical and digital siblings usually serves operational and planning purposes, and here, the key indicators are energy efficiency, asset performance, occupancy comfort and other user-defined measures. Figure 1 illustrates the overall architecture of the authors' digital twin framework. As indicated in the figure, the digital twin can be created from a physics-based model that takes into account system dynamics and interaction between its components. For instance, EnergyPlus, originally developed by the US Department of Energy (US DOE), models the thermal properties of a building

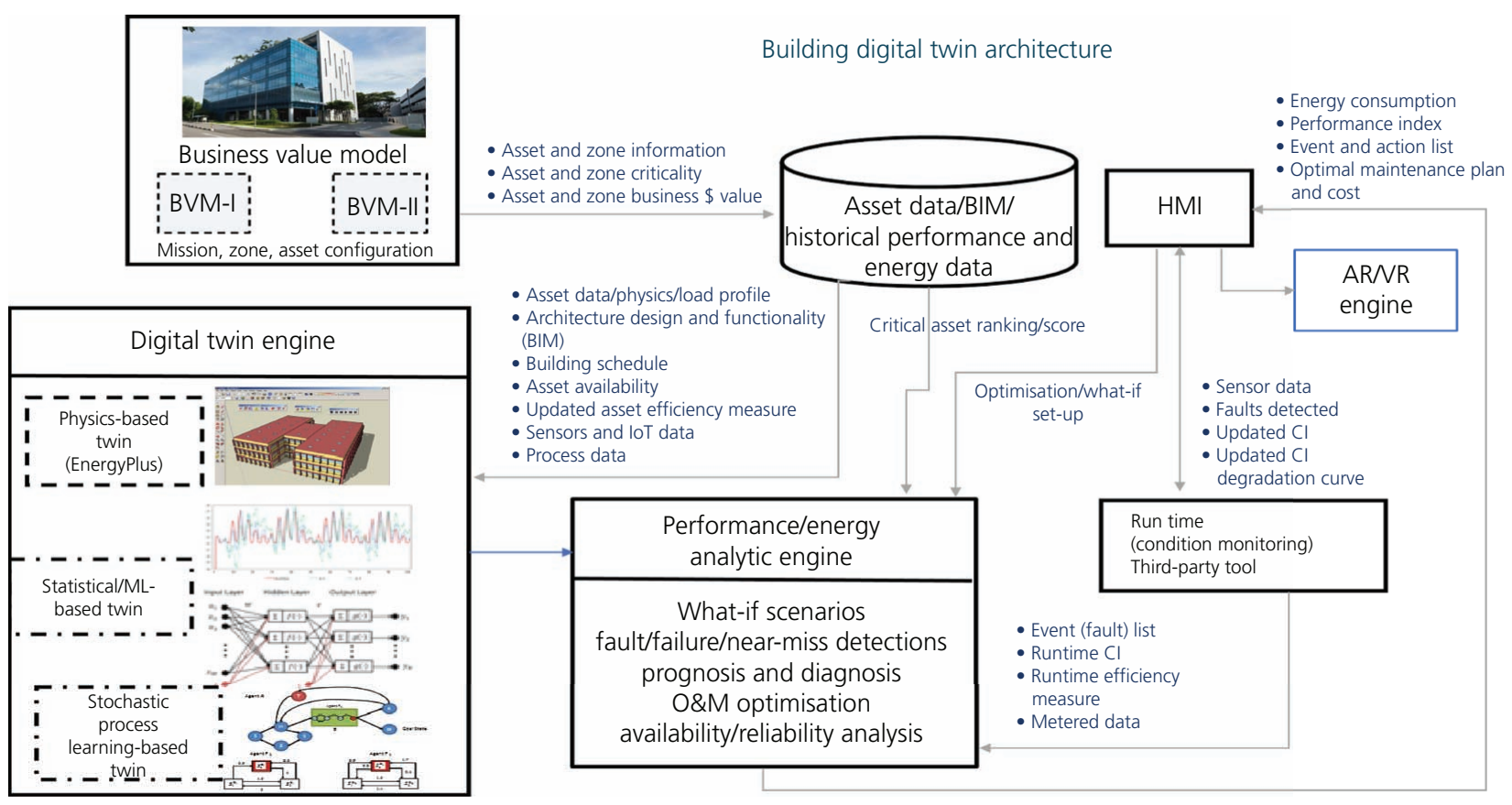

Figure 1. Digital twin architecture. AR, augmented reality; VR, virtual reality 
Smart Infrastructure and Construction

Volume 174 Issue 2
Improving building energy footprint and

asset performance using digital twin

technology

Jafari, Ghofrani, Zaidan and Abulibdeh and incorporates interactions between zones and its heating, cooling and ventilation assets. The impact of externalities, such as weather and occupancy, is also modelled. An EnergyPlus building model can be augmented with physics or artificial intelligence (AI)-based twins of assets that are not directly involved with building heating, ventilation and air conditioning (HVAC); examples are industrial machinery, air compressors, electric vehicles and other plug-ins. EnergyPlus simulation assumes a deterministic behaviour and does not incorporate random noises nor it does cover faults, failures and asset degradations. Furthermore, the simulation model does not allow for inputs during simulation runs; thus, live interactions between virtual and physical systems are not seamlessly possible. To take into account such stochastic behavioural patterns and sensory data feeds from physical assets, the authors include in their architecture an additional gateway module where random behavioural patterns (e.g. failure events and duration of failures) are modelled and communicated to the virtual model. In real life, the gateway would pass to the digital twin the occurrence and timing of real failure events.

Figure 1 architecture extends beyond EnergyPlus and allows for digital twins that are built using large data streams on asset conditions and behavioural patterns. In such cases, one can use statistical forecasting, artificial neural nets (ANNs) or similar computation/numerical models (Zhu et al., 2016) to link asset inputs to outputs and mine its behavioural patterns. Another option is to use a stochastic learning technique to develop a state/ transition model from process data and external signals. Unlike ANN-type models, process models (Farzan et al., 2015a) describe asset/process dynamics over an event-time horizon. In the absence of a physics-based model, a combined input/output and state/ transition model can serve as the strong digital twin of an asset or a system of assets. The integration with augmented reality and virtual reality will only be possible through a building information model (BIM) and in the presence of real-time asset condition data. Next, the authors will briefly describe the various modules of the architecture.

\section{Business value model of assets}

The term 'value' can broadly be defined in economic, social and/or environmental dimensions. A systematic methodology computes an asset's value derived from its business objectives. While such business objectives may remain constant for long periods of time (e.g. months or years), the value quantity may change dynamically. The authors use two types of asset business value models (BVM-I and BVM-II) to map the business objectives of buildings or groups of buildings to their assets (see Figure 2). BVM-I maps the business objectives of an organisation to its assets, using a four-step approach: (a) the relative importance among missions, $(b)$ the criticality of zones to missions, $(c)$ the impact of asset systems to zones and $(d)$ asset failure risk priority that takes advantage of failure mode and effect analysis. The result is the ordinal criticality of the asset. BVM-II quantifies the business value of assets in economic and monetary terms. Basically, it associates asset failures

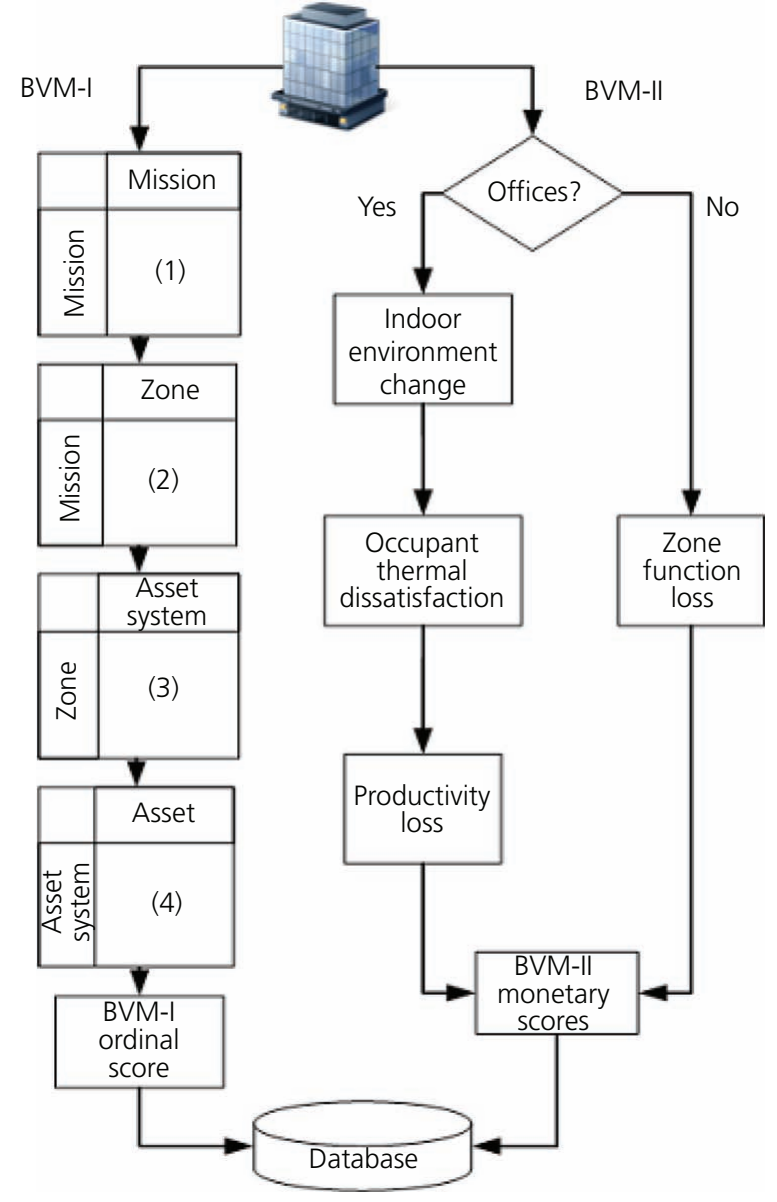

Figure 2. Business value model

with zone-functional losses, which can be quantified by occupants' thermal dissatisfaction and productivity losses. At the design stage, the performance characteristics of HVAC components are calculated to ensure that indoor air quality (IAQ) requirements are met. Here, the data requirement includes but is not limited to: missions/business objectives, functional and operational tasks, building zoning, assets of an HVAC system, lighting system, plug and industrial loads, occupancy profile, seasonal variation, indoor environment constraints, asset failure consequences and occupant income or income distribution, among others. For case studies and additional formulation, see Salahi et al. (2013).

\section{Digital twins}

Traditionally, digital twins were used to improve the performance of a single asset, for example, an engine, but more recently, they have been applied to the systems of assets or an entire organisation. As shown in Figure 1, the authors' digital twin engine covers several different types of models: physics based, statistics based, process based or a combination. A BIM provides the structural and informational foundation of a built environment, which, if integrated with a dynamical model, can yield a set of desired physical quantities. Combining the BIM model with 
Smart Infrastructure and Construction

Volume 174 Issue 2
Improving building energy footprint and asset performance using digital twin

technology

Jafari, Ghofrani, Zaidan and Abulibdeh
EnergyPlus yields the energy (power and natural gas) consumption of a building under different internal and external conditions. The combined model (see Figure 3 ) has a building's physical make-up (floors, zones and their interactions, building envelop and materials used, windows and so on), all associated mechanical systems (heating, cooling and ventilation and so on) and occupancy data and schedule. The model is capable of calculating the heating and cooling loads necessary to maintain thermal control setpoints, conditions throughout a secondary HVAC system and coil loads and the energy consumption of primary plant equipment. The extended combined model of a built environment can further cover all building accessories and equipment (e.g. air compressors, plug-ins and electric vehicles, $\mathrm{EVs}$, industrial or laboratory equipment) that consume energy or utilities, in general.

For illustrative purposes, consider a community of eight buildings and a shared energy storage. The buildings are US DOE reference buildings described in Table 1.

Figure 3 gives the high-level digital template of this community, where each building icon is linked to a BIM+EnergyPlus model (see Figure 4). With this digital model in hand, the authors can evaluate new HVAC setpoint controls under various weather and climate conditions. For instance, they consider three different climate zones: Illinois, New Jersey and Texas, to evaluate the

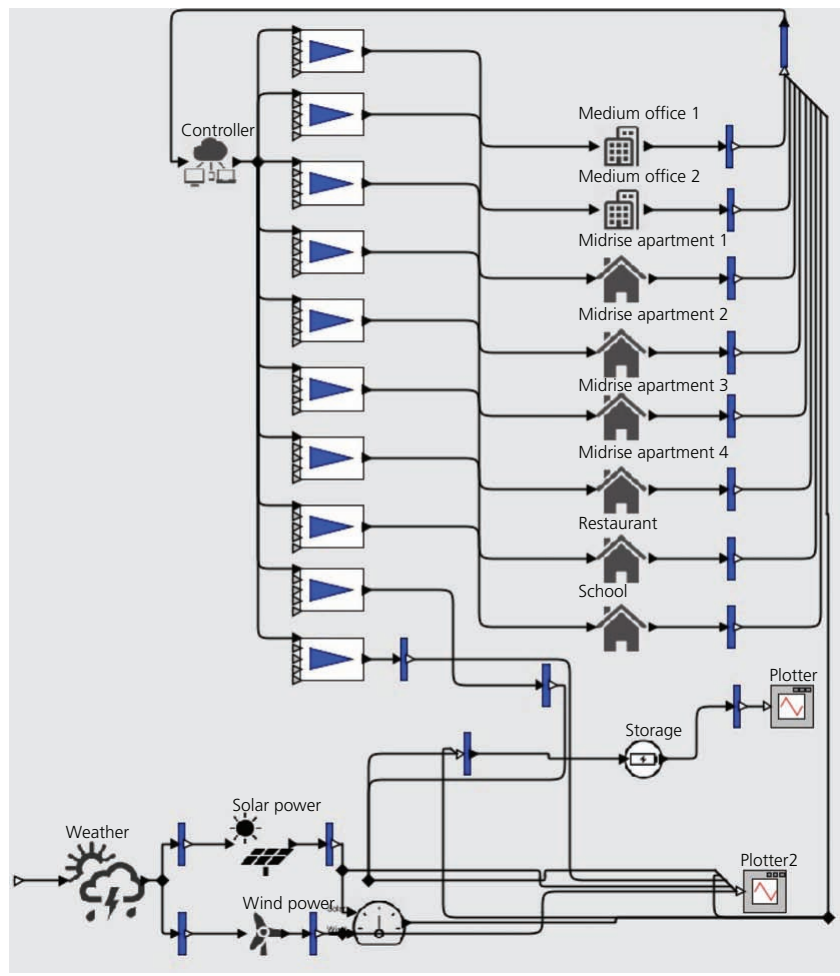

Figure 3. Schematic of the community
Table 1. Building simulation description

\begin{tabular}{|lccc|}
\hline Building type & $\begin{array}{c}\text { Number of } \\
\text { buildings }\end{array}$ & Area: $\mathbf{m}^{\mathbf{2}}$ & $\begin{array}{c}\text { Number of } \\
\text { zones }\end{array}$ \\
\hline Medium office & 2 & 4982 & 15 \\
Mid-rise & 4 & 3135 & 27 \\
$\quad$ apartment & 1 & 6871 & 25 \\
$\begin{array}{l}\text { Primary school } \\
\text { Service } \\
\text { restaurant }\end{array}$ & 1 & 511 & 3 \\
\hline
\end{tabular}

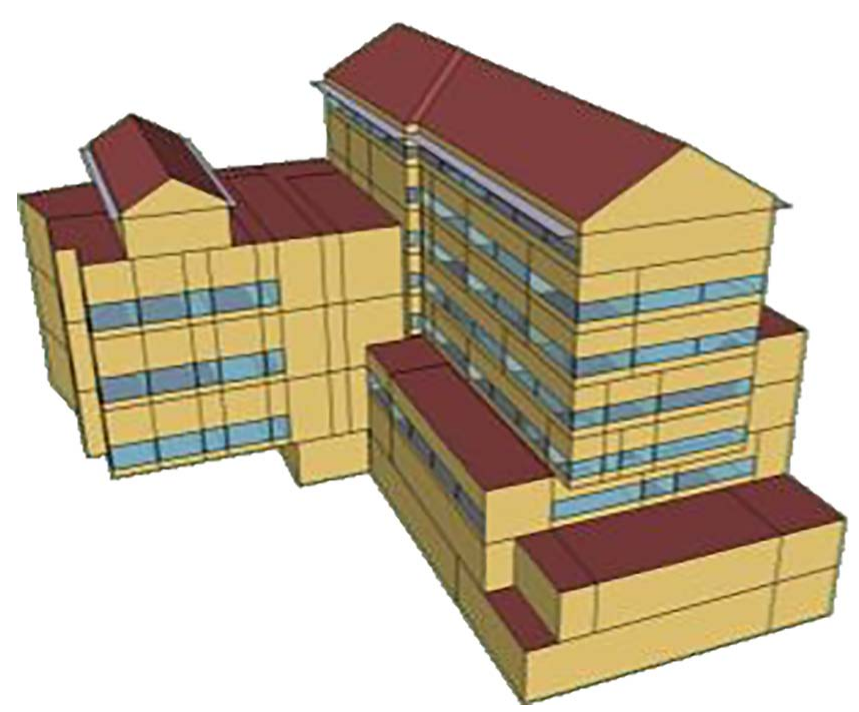

Figure 4. A building digital twin

impact of climate on the combined onsite generation and storage behaviour. It is assumed that the elevation and design of the renewable energy site and storage remain constant in the three scenarios. Also, all the buildings have the capability of adjusting their operation with different peak demands and the availability of onsite generation. The timestep for the simulation is $15 \mathrm{~min}$. Over these simulations, human occupancy patterns and plug loads can change. For detail formulation and discussion, see Ghofrani and Jafari (2017).

Defining a spectrum of models from purely data driven to purely physics based, the following prediction models for internal temperature and energy consumption fall closer to the left-hand side of the spectrum,

1. $T_{\text {in }}^{i+1}(i)=T_{\text {in }}^{i}(i)+\alpha_{i} \cdot R^{t}(i)+\phi_{i} \cdot\left(T_{i n}^{i}(i)-T_{\text {ext }}^{i}(i)\right)+\varepsilon^{t}$

where $T_{i n}^{i}(i)$ is the internal temperature for zone $i, T_{\text {ext }}^{i}(i)$ is the external temperature for zone $i, \alpha_{i}$ and $\phi_{i}$ are parameters and $R^{t}(i)$ is the power rate (Farzan et al., 2015b). Energy consumption is also defined by a data-driven model as a function of (current and past ambient temperature, current and past relative humidity, current and past occupancy, states of HVAC-related equipment, 


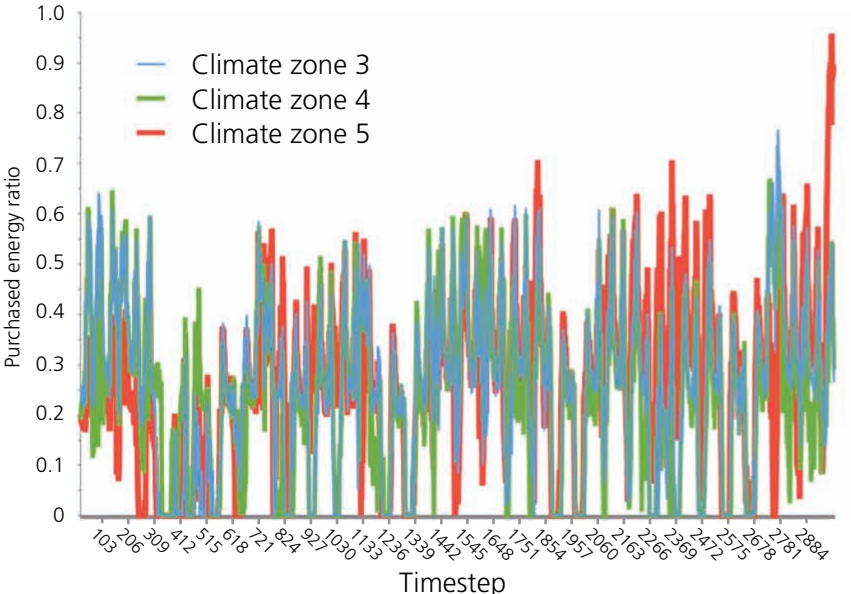

(a)

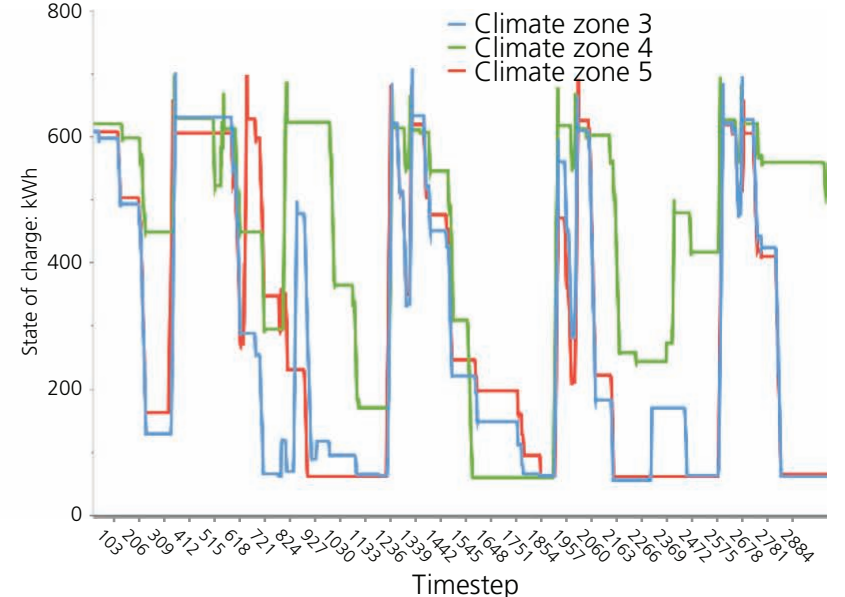

(b)

Figure 5. An illustration of how digital twins can be used for what-if analysis: (a) purchased energy ratio in different climate zones and (b) energy storage behaviour in three different climate zones

current and past set points). The predictive model for the energy consumption will then take the form described below with a regression part defined on weather and sensible cooling or heating rates and a time series part that takes the terms defined above. The authors have

$$
\begin{aligned}
y_{t}= & \sum_{j=0}^{K} \beta_{j} x_{t j}+\varepsilon_{t} \sum_{i=0}^{p} \sum_{j=0}^{P}(-1)^{i+j-1} \phi_{i} \phi_{j} B^{i+s \times j} \\
& +\sum_{i=0}^{q} \sum_{j=0}^{Q}(-1)^{i+j} \theta_{i} \Theta_{j} B^{i+s \times j}
\end{aligned}
$$

2.

This model performs well with prediction but is incapable of evaluating different design, operation or maintenance alternatives.

Towards the middle of the spectrum, the authors include models that combine physics and data-driven properties. These models are more suitable to analyse and evaluate design and operation alternatives. In particular, the authors use the model developed in Ghofrani et al. (2020), where they take into account the important factors in heat transfer phenomenon in a thermal zone, such as air distribution system, solar irradiation, outside air convection heat transfer, facing orientation, internal gains and infiltration and air mixing. A mixed data-driven model is used to predict a building's thermal behaviour. The prediction model covers a set of physicsbased independent variables, a set of categorical variables and some time series data (such as temperature in the previous time epochs). An example is shown in Figure 6 where the building indoor temperature response can be predicted for a variable air volume system under different airflows. The above model yields the dynamic thermal mapping of a building and its zones, which can be used to construct advanced distributed and on-demand control schemes for heating and cooling. This will be discussed later in this article. The thermal data feed to the following datadriven model to predict load $(Y)$ at time $t$,
3. $Y=h_{w}(X)$ and $\min \sum_{i=1}^{n}\left(Y_{i}-Y_{i}\right)^{2}+\Gamma(w, \alpha)$

where $Y$ is the predicted building load, $h_{\mathrm{w}}(X)$ is a hypothesis function with the model parameter $w$ and feature vector $X, Y_{i}$ is the real load for instance $i, n$ is the size of the training dataset, $\Gamma(w, \alpha)$ is a regularisation term in the cost function (meansquared error in this case). A challenging task here is to identify and collect data for the main attributes in the input feature vector $(X)$ to construct the predictor. The feature vector can comprise multiple independent factors such as lagged target values, weather condition, building schedule and categories such as time of day or weekend/weekday indicators. Figure 7 illustrates how a multivariate linear regressor with categorical variables can follow the simulated load of a building (see Ghofrani and Jafari, 2017).

In addition to the engineering or statistical approaches introduced above, the authors also take advantage of bottom-up approaches to construct energy load models (see Farzan et al., 2015b). Finally, use resistance-capacitance (RC) models can be used for building thermal modelling and analysis. Methodologies such as balanced truncation, lumped capacitance, the subspace method and complex RC models are among the applications of the RC approach (Belić et al., 2016; Goyal and Barooah, 2011; Liu et al., 2016).

\section{Analytic engine}

Besides the above operation-related analysis, the analytic engine includes modelling tools for the failure/fault analysis of building assets. The bathtub curve for the hazard rate function is assumed where the rates are conditioned on the life stage of an asset, namely, BOL (beginning of life), MOL (middle of life) and EOL (end of life). The use of the bathtub curve for HVAC equipment and its electrical and mechanical components is common in the 


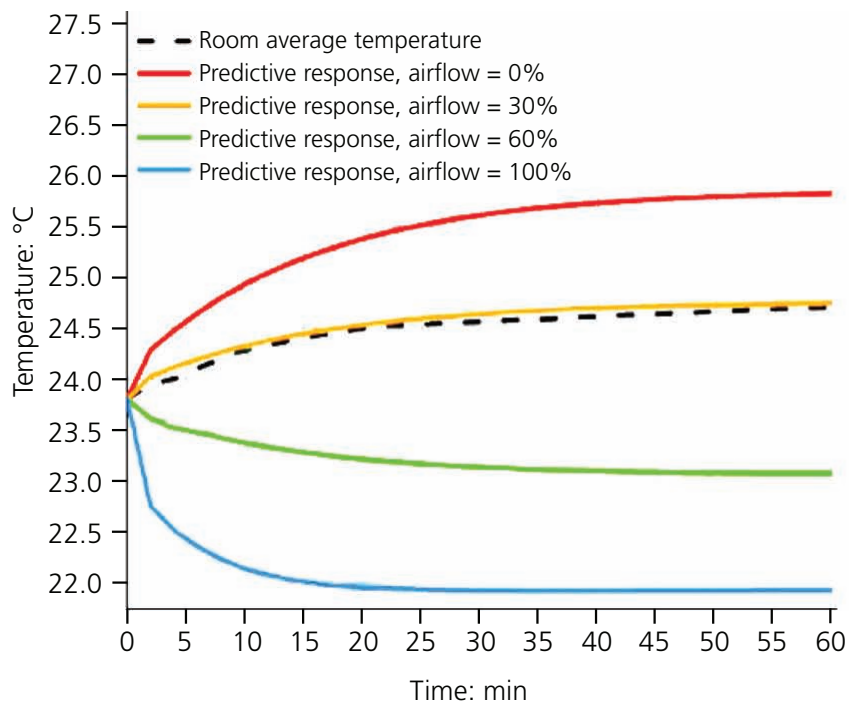

(a)

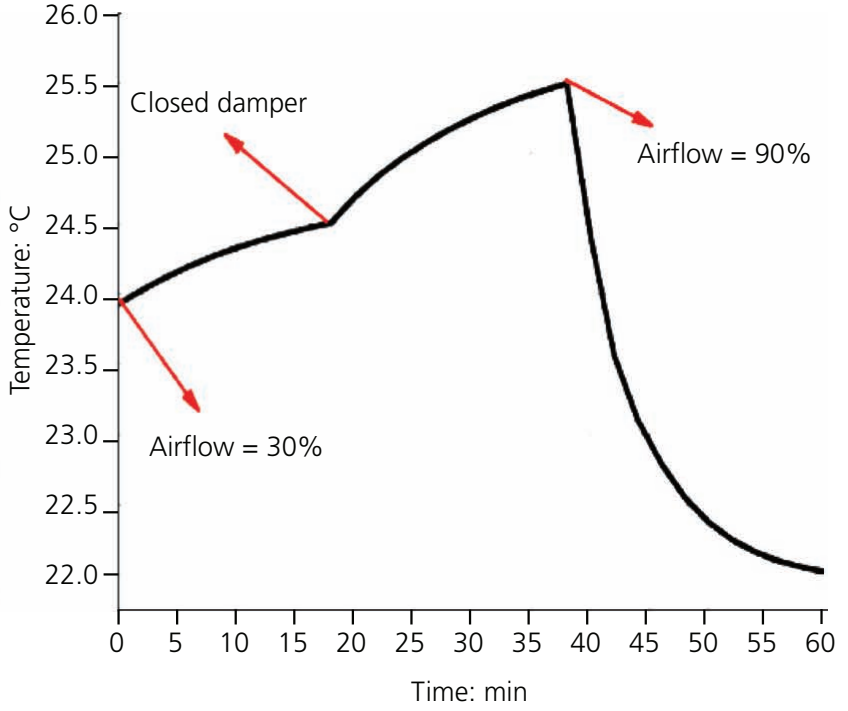

(b)

Figure 6. Prediction of building indoor temperature variation under different inlet airflows: (a) thermal behaviour under different airflows over a $1 \mathrm{~h}$ period and (b) thermal behaviour under different imposed airflows at different times

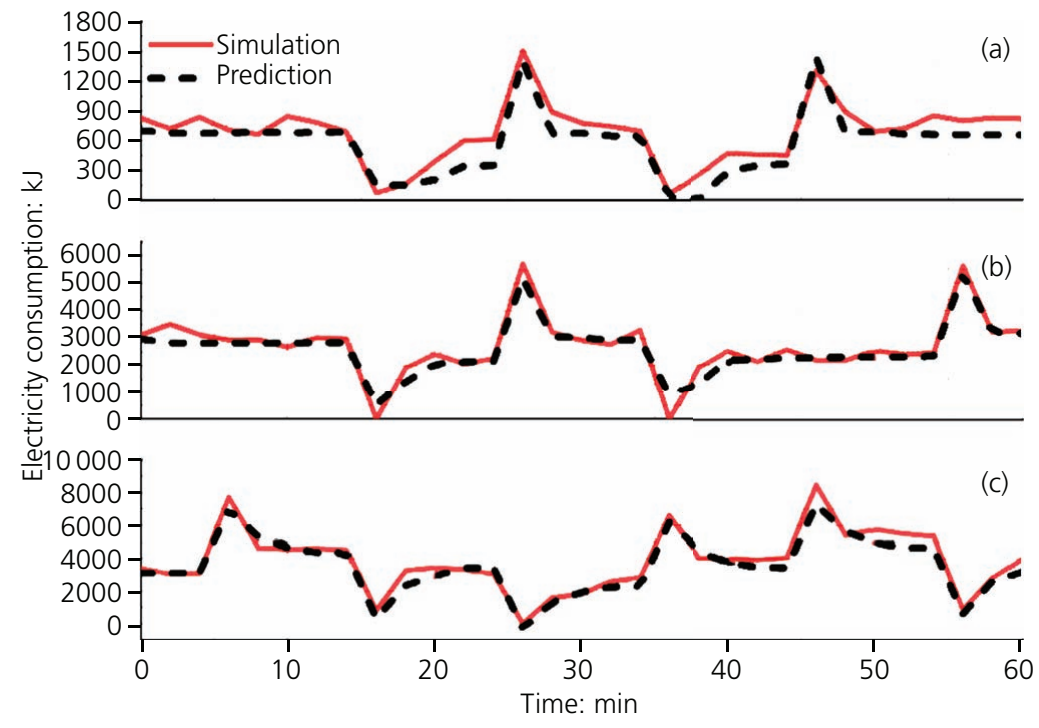

Figure 7. Linear regressor predicting simulated building load for three different buildings: (a) a small office simulation, (b) a medium office and (c) a large office

literature (Ghosh and Campell, 2004). The formulation also includes a restoration factor $(\mathrm{RF})$ for each maintenance and the real and effective ages for each asset. A relationship exists between an asset's real age and its effective age, depending on the maintenance actions (Hendron, 2006). This relationship includes an asset's condition index, $\mathrm{CI}_{i}$. In their framework, the authors extend the definition of a CI to also include its energy efficiency and the ratio of its nominal (expected) to actual power consumptions. The performance degradation of an asset can then be captured by quantifying the drop-in efficiency or increase in power consumption for a particular working condition. The framework also allows for monitoring the energy performance of building spaces measured at a zone level. The CI of a zone is defined as its energy use intensity (EUI), in kilowatt per hour per square foot per year, divided by the EUI of the best performing zone. Sensor and control values are used to perform estimations based on the physical properties of the system. Discrepancies of estimated and measured values are collected as a detection failure 
vector. The expected values are predicted from asset's digital twin. Diagnosis seeks to find the most probable cause for the observed failures. The system generates CI for the building asset detected with faults. This applies to general assets such as variable air volume boxes. The CIs are defined based on their faulty conditions, assuming a full-functioning asset with a CI as 100 , a totally failed asset with a CI as 0 and a faulty asset with a CI, depending on the fault type. The asset effective age (in hours) at each time step $h$ is quantified according to the relationship given by (Kahle, 2007; Kijima et al., 1988; Misra, 2008)

4. $A_{E f f_{i}}(h+1)=A_{E f f_{i}}(h+\mathrm{PLR}) \times\left(1-\mathrm{RF}_{(T k l)_{i}}\right)$

Note that $\mathrm{RF}_{(T k l)_{i}}$, is defined based on the type of maintenance action on asset $i$; it is set to zero if no maintenance is applied for period $T$. PLR $\in(0,1)$ is the part load ratio of the asset for specific time interval between $h$ and $(h+1)$. The asset loads or PLRs can be calculated from the EnergyPlus simulation. Keeping the real age of an asset fixed, its effective age improves by the maintenance actions and it is a common practice to approximate these shifts by an average smooth function over an asset's real life. The model assumes that there is a positive correlation between effective age of an asset and its energy efficiency. To optimise maintenance planning, the authors include maintenance cost, penalty cost obtained from BVM and asset energy and operational cost. An asset energy cost includes the fixed and variable costs of consuming or generating energy (e.g. electric energy and natural gas). The framework includes a multiobjective stochastic optimisation with a trade-off between capital expenditures and savings from energy and operations. A set of user-defined maintenance actions of reactive and proactive nature are included. The stochastic elements of the problem are load on assets (e.g. chiller's PLR and supply fan's flow) and asset failure events. Loads can be simulated based on building configuration, operation and controls and weather conditions. Failure events are defined by asset lifetime distributions, with bathtub hazard rates and Weibull functions. There is also random dependency between an assets' performances, shown by

5.

$$
\boldsymbol{\delta}(\boldsymbol{T} ; \boldsymbol{k}, \boldsymbol{l})=\left[\begin{array}{cccc}
\delta_{11} & \delta_{12} & \ldots & \delta_{1 n} \\
\delta_{21} & \delta_{22} & \ldots & \delta_{2 n} \\
\vdots & \vdots & \ddots & \vdots \\
\delta_{n 1} & \delta_{n 2} & \ldots & \delta_{n n}
\end{array}\right] \forall(T ; k, l)
$$

Note that the diagonal elements of the above matrix represent the direct impact of maintenance actions. $\delta_{11}$ is the shift in asset 1 's effective age as a result of maintenance option $(T ; k, l)$ and $\delta_{12}$ is the improvement in asset 2 's effective age as a result of exercising option $(T ; k, l)$ on asset 1 .
The analytic and digital twin engines run in parallel and communicate using a gateway. The probability of failures and energy performance degradation trend of an asset depend not only on the time elapsed since the asset's installation (actual age) but also on changes resulting from the cumulative load on the asset as well as the maintenance policies employed (effective age). The effective age of assets is input to the analytic engine at the beginning of every cycle (usually in hours). The engine then calculates the failure probability and energy performance efficiency of the assets as a function of their effective ages, and these values are then passed to the digital twin to calculate building energy consumption. The energy transfer or conversion efficiencies of assets are calculated based on their PLR. An asset's PLR is then computed by the digital twin and passed to the analytic engine for the calculation of degradation and maintenance optimisation. Random failure events, characterised by asset availabilities, are also generated based on probability distributions in the analytic engine. Asset performance and efficiency measures and availability indicators are then passed to the digital model. The analytic engine then updates the asset's effective age and CI according to the improvement factor of the type of maintenance policy specified. The data exchange between digital twin, analytic engine and BVM is made possible using gateways, which are software codes for data translation and formatting between various modules.

The analytic engine also includes constructs to optimise building operations with respect to energy consumption, occupancy comfort and asset performance. Generally speaking, the objective is to find an optimal decision variable vector, $\boldsymbol{X}^{*}$, to minimise cost, $\phi(X)$, and discomfort, $\psi(\boldsymbol{X})$, with respect to constraints associated with building physics, $\Pi_{i}(\boldsymbol{X})$, and other arbitrary constraints (e.g. asset availability, schedules), $\xi_{j}(\boldsymbol{X})$. The constraints can vary based on the behaviour and functionality of the building, while linearisation methodologies could also be applicable in constructing the optimisation framework.

$$
\begin{gathered}
\min \phi(X)+\psi(\boldsymbol{X}) \\
s . t . \Pi_{i}(\boldsymbol{X}) \leq 0, i \text { and } j=1,2,3, \ldots \\
\xi_{j}(\boldsymbol{X}) \leq 0, j=1,2,3, \ldots
\end{gathered}
$$

The decision variable vector $X$ can be control schemes and/or setpoints (e.g. temperature, humidity, lighting). The framework takes into account complex factors associated with the thermal behaviour of a building, building characteristics such as infiltration and construction materials, occupancy patterns, weather condition and so on, for a more realistic and effective decision making system to save cost and energy and enhance the occupants' comfort (see Ghofrani et al., 2019). A better understanding about the thermal behaviour and occupancy patterns at zonal level in a building can highly improve operation control for air condition and lighting systems. Once the 
occupancy patterns for each zone (e.g. the time of arrival/departure, the number of people) are captured by way of deterministic schedules or data-driven approaches, preoccupied and post-occupied planning schemes can be utilised to avoid unnecessary air conditioning and lighting. Once the arrival time of an occupant is determined, the cooling/heating system can be initiated at an optimal time to achieve comfort levels on time and maintain the environment in the comfort zone until the zone is occupied. This needs computing the zonal thermal response under different externalities (by way of simulation, regression and other computational methods) to be able to project temperature and humidity variations in the future. As an example, a control strategy can be constructed as in Figure 8
(Ghofrani and Jafari, 2017), where the start and shutdown times for cooling are synchronised to expected occupancy based using a zone's thermal map and external conditions, such as temperature, wind and sun exposure. The timescale and period for control settings is defined by the user (this example is set to $10 \mathrm{~min}$ ).

\section{Concluding remarks}

This paper contributes by integrating the existing building digital twinning and optimisation techniques into a framework for the purpose of optimising building energy efficiency over a range of asset maintenance and operation planning alternatives. The traditional use of digital twin technology at the design stage was extended to the

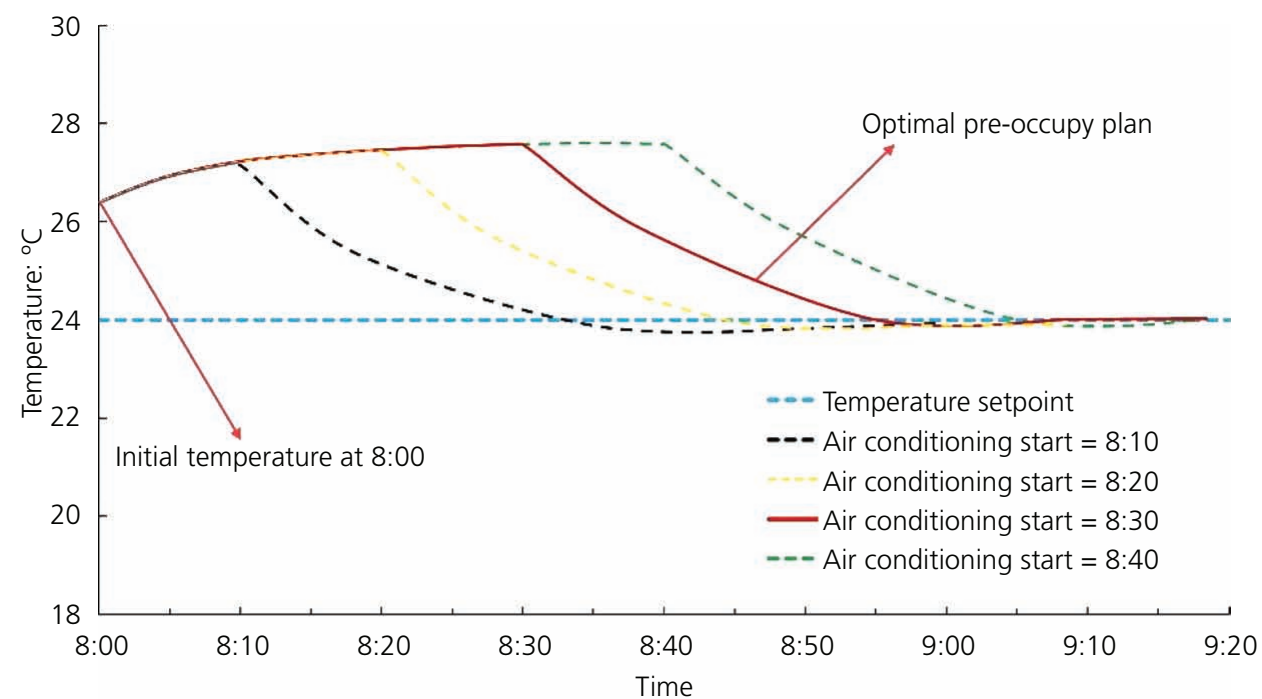

(a)

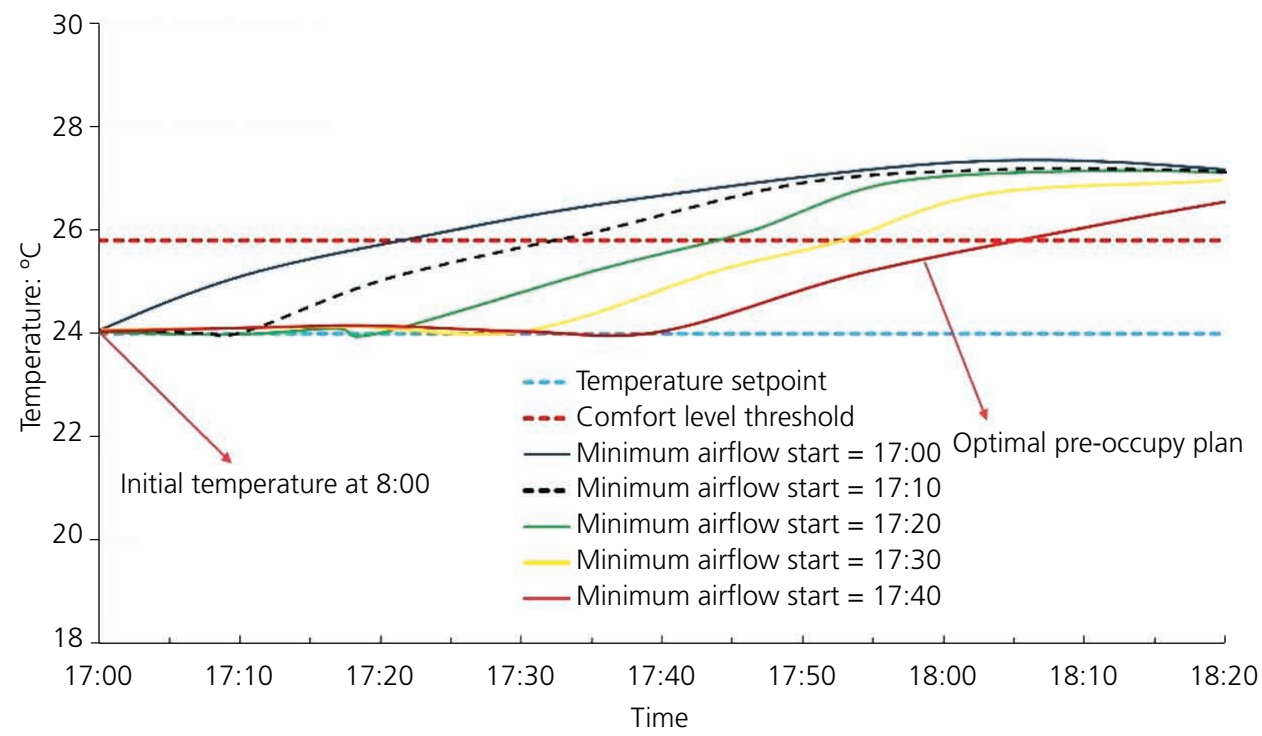

(b)

Figure 8. (a) Pre-occupancy synchronisation of cooling and (b) post-occupancy synchronisation of cooling 
Smart Infrastructure and Construction

Volume 174 Issue 2
Improving building energy footprint and

asset performance using digital twin

technology

Jafari, Ghofrani, Zaidan and Abulibdeh maintenance planning and operation control of building assets. Digital twinning was defined using three-dimensional digital models, statistical models with time series and regression components and/or hierarchical process models. The combined digital twin incorporates different aspects of building physics, for example, thermal behaviour, external conditions (e.g. temperature and humidity) and occupancy characteristics (e.g. preferences and the time of use). The framework allows for the integration of internet of things (IoT) data from building assets in real time, mainly used for updating the forecasts and comparing actual assets' condition and energy use data to data from IoT devices. This can provide valuable information for prognosis and planning appropriate maintenance or operational actions. O\&M optimisations take advantage of functional or business values that building assets generate, using the existing BVM models.

\section{Acknowledgments}

This publication was made possible by partial support from NPRP grant number NPRP11S-1228-170142 and number NPRP13S-0206200272 from the Qatar National Research Fund (a member of Qatar Foundation). The findings achieved herein are solely the responsibility of the authors.

\section{REFERENCES}

Barton R (2002) Strategic asset management incorporating ecologically sustainable development. Journal of Facilities Management 1(1): 70-84.

Belić F, Hocenski Z and Slišković D (2016) Thermal modeling of buildings with RC method and parameter estimation. In 2016 International Conference on Smart Systems and Technologies (SST). IEEE, Piscataway, NJ, USA, pp. 19-25.

Chin W and Gillepsie J (2010) Breaking down the silos between energy and asset management: asset performance optimization. Hydrocarbon Processing 89(3): 4-35.

Farzan F, Jafari MA, Gong J, Farzan F and Stryker A (2015a) A multi-scale adaptive model of residential energy demand. Applied Energy 150: 258-273.

Farzan F, Vaghefi SA, Mahani K, Jafari MA and Gong J (2015b) Operational planning for multibuilding portfolio in an uncertain energy market. Energy and Buildings 103: 271-283.
Ghofrani A and Jafari MA (2017) Distributed air conditioning control in commercial buildings based on a physical-statistical approach. Energy and Buildings 148: 106-118.

Ghofrani A, Farzan F, Swartz J et al. (2016) Cyber physical simulation of energy smart communities. In Transforming the Future of Infrastructure through Smarter Information: Proceedings of the International Conference on Smart Infrastructure and Construction, 27-29 June 2016. ICE Publishing, London, UK, pp. 663-667.

Ghofrani A, Nazemi SD and Jafari MA (2019) HVAC load synchronization in smart building communities. Sustainable Cities and Society 51: 101741

Ghofrani A, Nazemi SD and Jafari MA (2020) Prediction of building indoor temperature response in variable air volume systems. Journal of Building Performance Simulation 13(1): 34-47.

Ghosh D and Campell R (2004) HVAC equipment aging and reliability issues at commercial nuclear power plants. In 28th Nuclear Air Cleaning and Treatment Conference, Albuquerque, NM, USA.

Goyal S and Barooah P (2011) A method for model-reduction of nonlinear building thermal dynamics. In American Control Conference on O'Farrell Street, 2077-82. San Francisco, CA, USA.

Hendron R (2006) Building America Performance Analysis Procedures for Existing Homes. National Renewable Energy Lab, Golden, CO, USA.

Kahle W (2007) Optimal maintenance policies in incomplete repair models. Reliability Engineering \& System Safety 92(5): 563-565.

Kijima M, Sumita U and Suzuki Y (1988) Periodical replacement problem without assuming minimal repair. European Journal of Operational Research 37(2): 194-203.

Liu W, Wang H, Zhao H et al. (2016) Thermal modeling for energy efficient smart building with advanced overfitting mitigation technique. In 201621 st Asia and South Pacific Design Automation Conference (ASP-DAC). IEEE, Piscataway, NJ, USA, pp. 417-422.

Lounis Z and Vanier DJ (2000) A multiobjective and stochastic system for building maintenance management. Computer-Aided Civil and Infrastructure Engineering 15(5): 320-329.

Mahani K, Jafari MA, Salahi N, Lu Y and Zhu J (2014) Optimizing building energy footprint using integrated reliability and energy plus simulation approach. ASHRAE Transactions 120(2): 416-429.

Misra KB (2008) Handbook of Performability Engineering. Springer, London, UK.

Salahi N, Jafari MA, Zhu J, Mahani K and Lu Y (2013) Business value driven asset management. ASHRAE/Ttransactions 121(2).

Zhu J, Vaghefi SA, Jafari MA, Lu Y and Ghofrani A (2016) Managing demand uncertainty with cost-for-deviation retail pricing. Energy \& Building 118: 46-56.

\section{How can you contribute?}

To discuss this paper, please email up to 500 words to the editor at journals@ice.org.uk. Your contribution will be forwarded to the author(s) for a reply and, if considered appropriate by the editorial board, it will be published as discussion in a future issue of the journal.

Proceedings journals rely entirely on contributions from the civil engineering profession (and allied disciplines). Information about how to submit your paper online is available at www.icevirtuallibrary.com/page/authors, where you will also find detailed author guidelines. 